\title{
Soil-fluid-structure interaction applied to the Oued Taht dam (taking into account the membrane effect).
}

\author{
Krenich Nasreddine ${ }^{1}$, Tahar Berrrabah Amina ${ }^{1}$, Houmadi Youcef ${ }^{1}$, Belharizi Mohamed ${ }^{2}$, Mehdeli Mohamed ${ }^{1}$, \\ Mohammedi Mohamed el Amine ${ }^{1}$ \\ ${ }^{1}$ Centre universitaire Belhadj Bouchaib, Laboratoire des structures Intelligentes, Département de Génie Civil, Ain \\ Témouchent, Algérie. \\ 237 Impasse Armand, 92160 Antony, France
}

\begin{abstract}
The objective of this work is to analyze the dynamic behavior (modal behavior) of the "Oued Taht" arch dam located at MASCARA, taking into consideration the effect of soil-fluid-structure interaction. The finite element code "Ansys" was chosen for the dynamic modeling of the dam that is the subject of this study. Three hypotheses were used for soil-structure interaction modeling; model with embedded base which corresponds to the case where the phenomenon of interactions soil-structure is neglected, model with ground of foundation without mass which consists in taking into account the kinematic component of interaction soil structure and neglecting the inertial component and the model with foundation soil with mass where the two components of soil-structure interaction are taken into account. For the fluid, the model of added masses (equivalent to the westergaard approach) using the SURF element available in the Ansys code library was used. A comparison between the different models of the "Oued Taht" dam was made; it has been found that the taking into account of the soil-fluid-structure interaction phenomenon modifies the period of the system and that the modeling of the dam with and without fluid gives a very important difference of the periods. The results obtained were compared with those of the "Brezina" dam, which is a gravity dam located in BAYADH. The work has shown that the periods of the "Oued Taht" dam with soil-fluid-structure interaction modeling are very out of phase with the periods without fluid modeling (taking into account only the soilstructure interaction phenomenon). which is not the case for the Brezina dam where the periods for the two models are getting closer. The periods between the two models mentioned before are close to the dam of Brézina because the latter is a dam which participates much more by its own weight than by its vault (thickness of the vault varies between $36.3 \mathrm{~m}$ at the base and $5 \mathrm{~m}$ in crest) which is the opposite for the dam "Oued Taht" which participates by its vault (constant thickness of $7 \mathrm{~m}$ ) thus the membrane effect is present, which is translated by the shift of the periods between the empty case and the filled case.
\end{abstract}

\section{Introduction}

La réponse d'une structure durant un séisme dépend des caractéristiques du signale sismique, du sol de fondation et de la structure elle-même. Le mouvement de la fondation est généralement différent du mouvement en champ libre (la difference du mouvement dépend de la différence de rigidité entre le sol et la fondation), cela est dû au couplage sol-structure durant le séisme, ce phénomène est connu dans la littérature sous le nom «d'interaction sol-structure ». Dans les structures où le domaine fluide existe, le phénomène devient plus compliqué; il s'agit «d'interaction sol-fluide-structure », les barrages en béton font partie de ce phénomène.

L'abondance de la littérature concernant l'interaction sol-structure traduit à la fois la complexité du phénomène et l'intérêt qu'y ont porté nombre de chercheurs [1], [2], [3].

On considère l'interaction sol-structure dans le but d'inclure dans les modes de déformations, les mouvements à la base de la structure, ainsi que pour obtenir une meilleure approximation de la période de vibration du système couplé qui sera plus long que la période du système avec base rigide. Dans la littérature, ils existent trois modèles pour la modélisation du phénomène d'interaction sol-structure: le modèle de la structure à base encastré, le modèle de la structure avec fondation modélisée sans tenir en compte sa masse, et le modèle de la structure avec fondation tenant en compte sa masse. 
Par rapport à la modélisation d'interaction fluidestructure, on peut citer trois approches: approche de Westergaard, l'approche de Lagrange et celle d'Euler.

L'objectif du present article est d'effectuer une étude paramétrique pour voir l'effet du phénomène d'interaction sol-fluide-structure sur le comportement du barrage voûte «Oued TAHT» don't l'existence de l'effet membrane. Pour la modélisation des différents cas du barrage en questions, le code en éléments finis ANSYS a été choisi.

\section{Présentation du cas d'étude}

Barrage Oued TAHT est un barrage voûte en béton, en cours d'exécution, il se situé dans la wilaya de Mascara en Algérie. Les caractéristiques mécaniques du corps du barrage et du sol de fondation ont été données dans le tableau 1.

Tableau 1. Propriétés des matériaux de barrage Oued TAHT

\begin{tabular}{|c|c|c|c|}
\hline Matériau & $\begin{array}{c}\text { Module de } \\
\text { young N/m2 }\end{array}$ & $\begin{array}{c}\text { Coefficient de } \\
\text { poisson }\end{array}$ & $\begin{array}{c}\text { Densité } \\
\mathbf{K g} / \mathbf{m} \mathbf{2}\end{array}$ \\
\hline $\begin{array}{c}\text { Corps de } \\
\text { barrage }\end{array}$ & $28.5 \mathrm{e}+09$ & 0.25 & 2500 \\
\hline $\begin{array}{c}\text { Sol de } \\
\text { fondation }\end{array}$ & $6.22 \mathrm{e}+09$ & 0.25 & 2100 \\
\hline
\end{tabular}

\section{Modélisation ansys d'interaction sol- fluide-structure du barrage oued taht}

L'effet d'interaction sol-fluide-structure sur le comportement du barrage Oued Taht a été modélisé et étudié utilisant le code ANSYS. Les résultats ont été discutés ci-dessous.

\subsection{Modélisation de l'interaction sol- structure}

La première application consiste à modéliser l'interaction sol structure, considérant le barrage vide, à cet effet trois hypothèses ont été adoptées :

Barrage à base fixe (encastrement).

Barrage avec sol de fondation, tenant compte de la masse de cette fondation et par conséquent de la composante inertielle de l'interaction.

Barrage avec sol de fondation, négligeant la masse de cette fondation et par conséquent de la composante inertielle de l'interaction.

\subsubsection{Modélisation en éléments finis du barrage}

L'élément type solid186 [4] disponible dans la bibliothèque du code ANSYS a été utilisé pour modéliser le corps du barrage ainsi que le sol de fondation.
La figure 1 représente le modèle du barrage à base encastrée (c à d sans tenir en compte le phénomène d'interaction sol-structure).

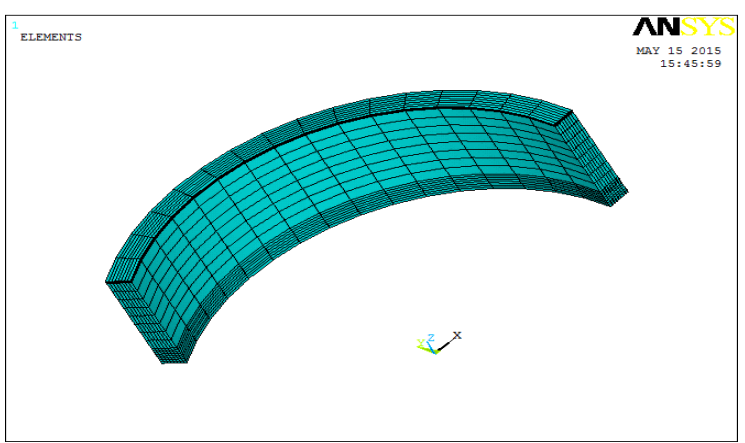

Fig. 1. Modèle élément finis du barrage à base encastré

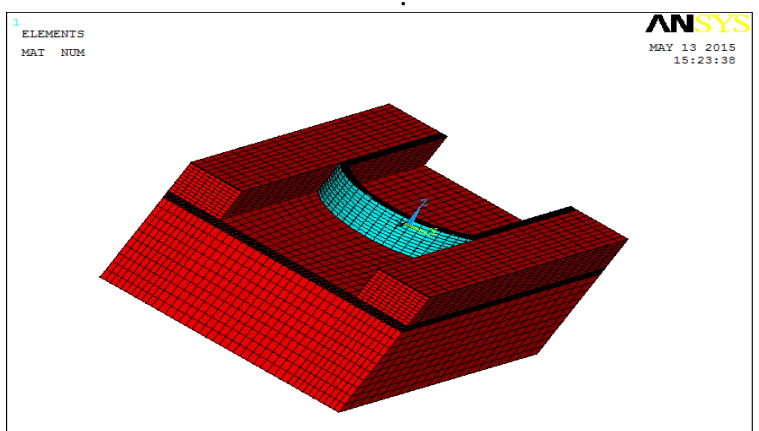

Fig. 2. Modèle élément finis du barrage avec sol de fondation.

La figure 2 représente le modèle du barrage avec sol de fondation; les dimensions du sol suivant $\mathrm{x}, \mathrm{y}$ et $\mathrm{z}$ ont été choisies après une étude de sensibilité du modèle aux dimensions du sol. Les extensions du sol de fondation sont comme suit :

Suivant x : $50 \mathrm{~m}$ de part et d'autre des extrémités du corps de barrage

Suivant y : $176 \mathrm{~m}$ de côté aval et amont de l'ouvrage

Suivant $\mathrm{z}$ : une extension de $-100 \mathrm{~m}$ a été choisie,

Il faut signaler que les extrémités du système ainsi que sa base sont encastrées.

\subsubsection{Effet d'interaction sol structure sur le comportement modal du barrage Oued Taht}

La figure 3, 4 et 5 schématise respectivement le mode fondamentale du barrage seul (à base encastré), barrage avec sol sans masse (non pesant) et barrage avec sol avec masse (pesant).

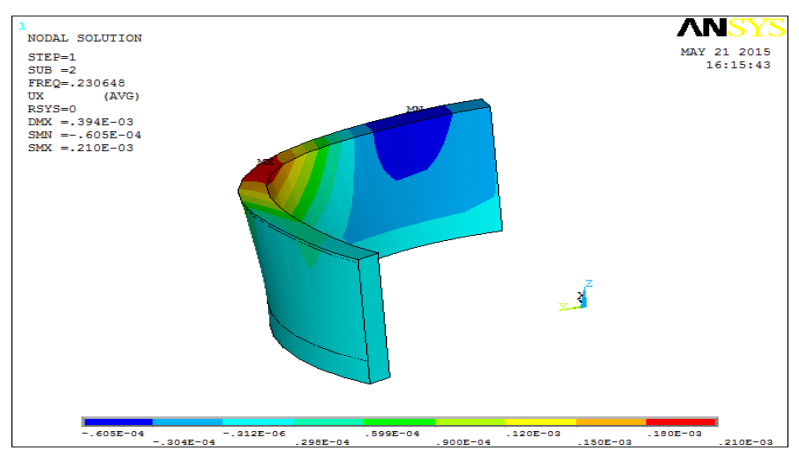

Fig.3. Schéma du mode fondamentale du barrage seul (mode 2) 


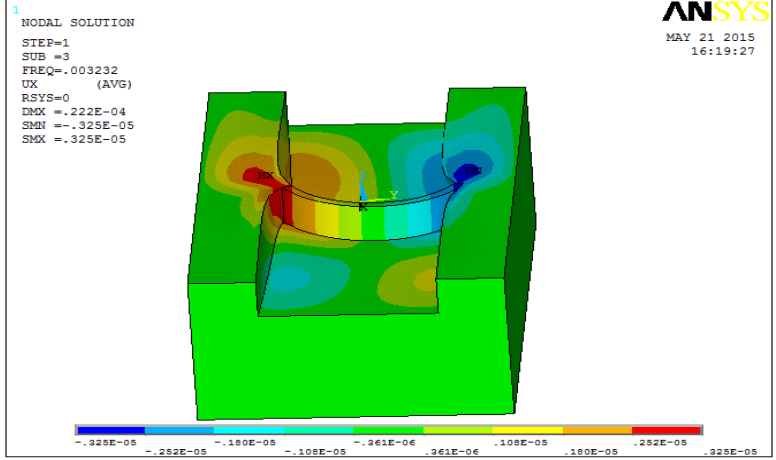

Fig.4. Schéma du mode fondamentale du barrage avec sol sans masse (mode 3 )

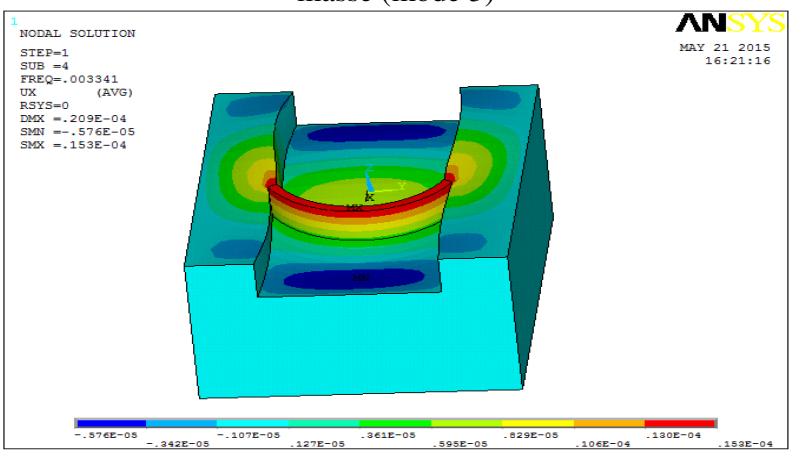

Fig.5. Schéma du mode fondamentale du barrage avec sol avec masse (mode 4)

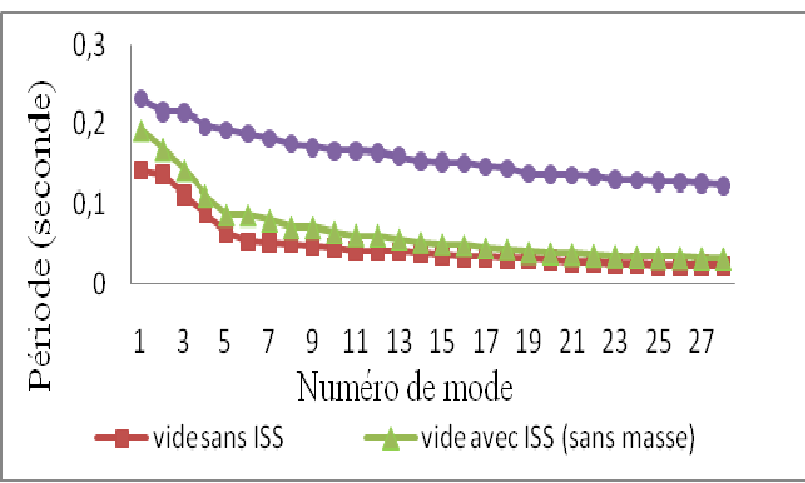

Fig. 6. Effet d' interaction sol-structure sur le comportement modal de barrage Oued Taht

La figure 6 représente une comparaison entre les premières périodes pour chaque modèle, il est à noter que le nombre de modes est choisi de telle façon que la somme des masses entrainées par ces modes atteints les $90 \%$ de la masse totale. Il est clair sur la figure 6 que la période du barrage augmente lorsque le sol de fondation est pris en compte, beaucoup plus lorsque le sol de fondation est modélisé avec sa masse. Les même résultats ont été trouvé pour le barrage poids voûte de el Bayadh [5].

Ajouter le sol de fondation à la structure, rend le système flexible et augmente sa masse ce qui engendre une diminution de la fréquence de système et par conséquent une augmentation de sa période.

Suivant les trois figures: 3 , 4 et 5 , on remarque que la modélisation du sol de fondation modifie non seulement les valeurs des périodes mais aussi le positionnement du mode fondamentale (sachant que le mode fondamentale est le mode qui entraine le maximum de masse). Les mêmes résultats sont trouvés pour le barrage d'el Bayadh [5], [6].

\subsection{Modélisation de l'interaction fluide structure}

La deuxième application consiste à modéliser l'interaction fluide structure, à cet effet le barrage est considéré plein. Pour chaque modèle d'interaction solstructure, deux analyses ont été mené avec et sans fluide. L'approche des masses ajoutées a été utilisée pour modéliser le fluide, En utilisant le code ANSYS, l'élément SURF154 disponible dans la bibliothèque ANSYS permet de représenter cette approche. Il s'agit de répartir la masse du fluide sur la surface de contact entre le réservoir et le barrage et/ou sol de fondation.

L'épaisseur de l'élément surf 154 est donnée par l'équation 1:

Epaisseur d'élément SURF154 = (B/A) x $\rho$

$A=$ surface de contacte fluide - barrage et/ou fondation (m2)

$\mathrm{B}=$ volume d'eau de réservoir $(\mathrm{m} 3)$

$\rho=$ masse volumique d'eau $(\mathrm{Kg} / \mathrm{m} 3)$

\subsubsection{Effet d'interaction fluide structure sur le comportement modal du barrage oued Taht}

Les figures 7,8 et 9 schématisent respectivement le mode fondamentale du barrage seul (à base encastrée), barrage avec sol sans masse et barrage avec sol avec masse tenant en compte la présence du fluide.

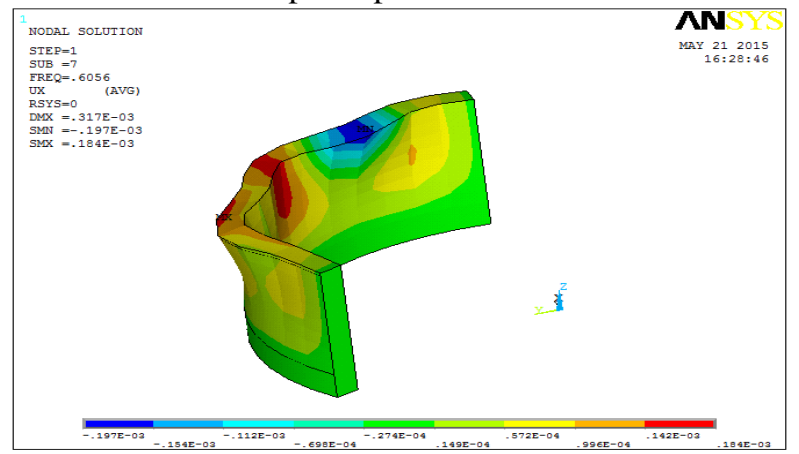

Fig.7. Schéma du mode fondamentale du barrage seul rempli (mode 7)

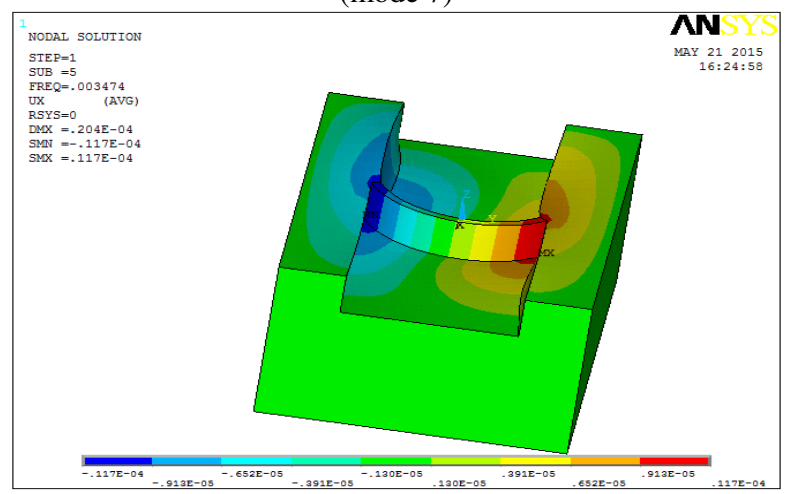

Fig. 8. Schéma du mode fondamentale du barrage avec sol sans masse rempli (mode 3) 


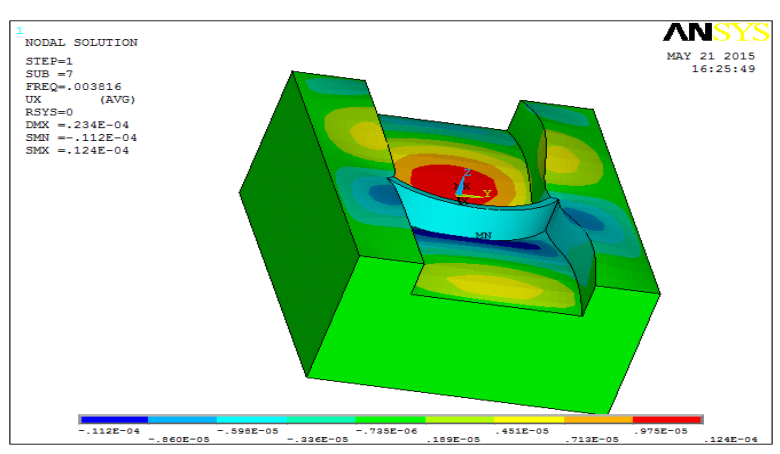

Fig. 9. Schéma du mode fondamentale du barrage avec sol avec masse rempli (mode 5)

\subsubsection{Barrage seul rempli}

La figure 10 présente l'effet de la modélisation d'interaction fluide structure sur le comportement modal du barrage seul c'est à dire sans modélisation d'interaction sol-structure.

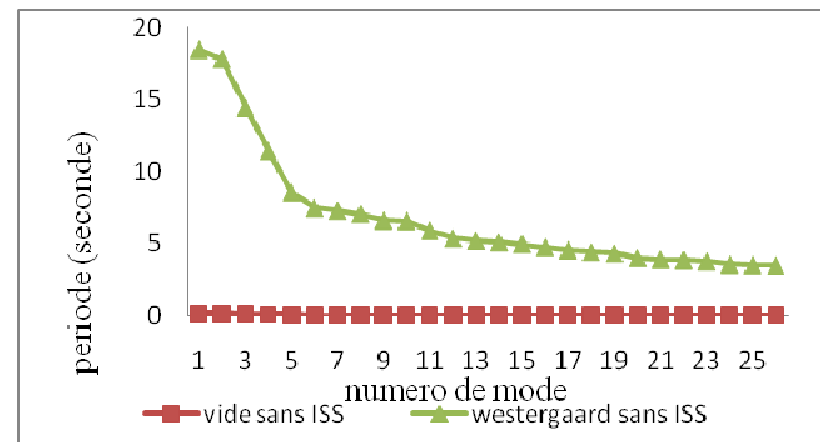

Fig. 10. Effet d'interaction fluide-structure sur la réponse modal du barrage seul (sans interaction sol-structure)

Il est clair sur la figure 10 que la présence du fluide augmente la période du système.

\subsubsection{Le barrage avec sol sans masse (rempli)}

Les mêmes résultats que ceux du barrage à base fixe ont été trouvés pour le cas du barrage modélisé avec sol de fondation sans masse.

\subsubsection{Le barrage avec sol avec masse (rempli)}

Les mêmes résultats que ceux du barrage à base fixe et du barrage modélisé avec sol de fondation sans masse ont été trouvés pour le cas du barrage modélisé avec sol de fondation sans masse.

Il a été constaté que la prise en compte du fluide augmente la période de la structure, c'est les mêmes résultats trouvés pour le barrage à base encastré et le barrage avec sol sans masse. Les mêmes résultats sont trouvés pour le barrage d'el Bayadh [7], la seul différence c'est que la présence du fluide pour ce barrage à un effet faible sur son comportement modale, cela est dû à la géométrie du barrage, c'est un barrage poids voûte, alors que pour le cas de barrage oued Taht, est un barrage voûte, ce qui implique l'effet membrane est présent dans son comportement. L'effet membrane se traduit par la différence de période entre le cas vide et le cas rempli.

\subsection{Comportement modal du barrage tenant en considération l'interaction sol-fluide- structure}

La figure 11 représente l'effet d'interaction sol-structure et de la présence du fluide sur le comportement modale du barrage Oued Taht, elle montre que la modélisation d'ISS ainsi que celle du fluide ont le même effet sur le comportement modal du barrage, c'est d'augmenter la période du système.

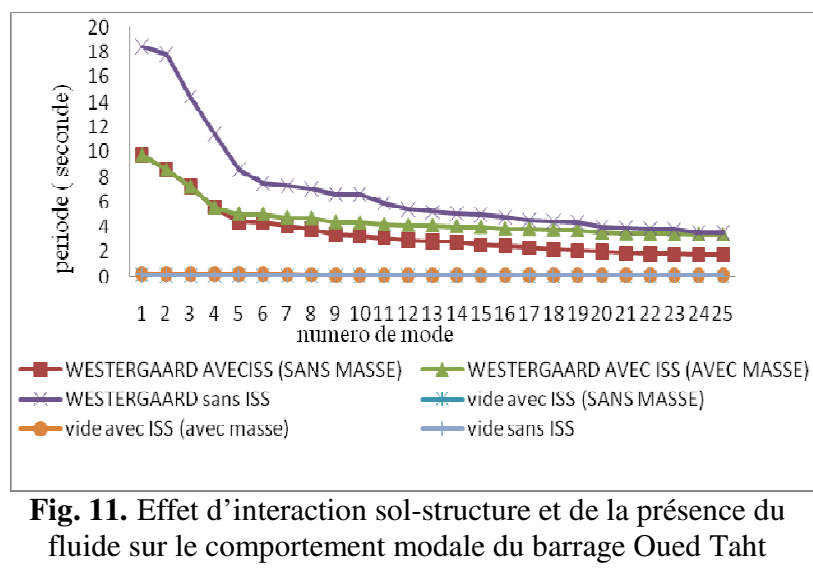

\section{Conclusion}

Dans cet article, une étude paramétrique a été menée pour le barrage voûte «Oued Taht» utilisant le code élément finis «ANSYS» pour voir l'effet du phénomène d'interaction sol-fluide-structure sur le comportement modale du système. L'analyse modale a permet de conclure que :

La modélisation d'interaction sol structure, modifie la période du barrage, sachant que la période est une caractéristique dynamique à prendre en considération dans les différents types de calcul dynamique (spectrale, transitoire...)

La modélisation d'Interaction fluide structure, modifie aussi la période du barrage.

La modélisation d'interaction sol structure et fluide structure donne une augmentation des périodes du système.

La grande différence de période entre le cas vide et le cas rempli confirme l'effet membrane du barrage Oued Taht, ce qui est n'est pas le cas pour le barrage de Brezina.

\section{Bibliographie}

1. LYSMER J. Analytical procedures soil dynamicsstate of the ASCE-conference on soil dynamics and earthquake engineering (1978).

2. IDRISS J.M and KENNEDY R.P.analysis for soilstructure interaction effects for nuclear power 
plants-report (by the AD HOC group, soil structure interaction of the committee on nuclear structures and materials of the structural division of ASCE, 1980).

3. ALAIN PEKER. Dynamique des sols (presses de l'école nationale des ponts et chaussées).

4. MANUEL. ANSYS Version 11.0 (2007).

5. A. TAHAR BERRABAH al. Three-Dimensional Modal Analysis of Brezina Concrete Arch Dam, Algeria, Earth Science Research; Volume. 1, No. 2. ISSN 1927-0542 E-ISSN 1927-0550. (Published by Canadian Center of Science and Education, 2012).

6. A. TAHAR BERRABAH al. Dynamic Soil Structure Interaction Study. (Jordan Journal of Civil Engineering, Volume 6, No. 2, 2012).

7. A. TAHAR BERRABAH al. Fluid-structure interaction of Brezina arch dam: (3D modal analysis, Engineering Structures 84, 2015)1928. 